\title{
A new receptor tyrosine kinase inhibitor, icotinib, for patients with lung adenocarcinoma cancer without indication for chemotherapy
}

\author{
XIAO ZHENG ${ }^{1 *}$, GUAN LIU $^{1 *}$, SHENGYE WANG $^{1}$, YUNLI ZHANG ${ }^{2}$, WENLONG BAO $^{3}$, \\ DEHOU DENG ${ }^{3}$, WEIMING MAO ${ }^{2,4}$ and MEIYU FANG ${ }^{3}$
}

\begin{abstract}
Departments of ${ }^{1}$ Radiation Oncology, ${ }^{2}$ Surgical Oncology, ${ }^{3}$ Integrated Chinese Traditional Medicine and Western Medicine, and ${ }^{4}$ Zhejiang Thoracic Oncology Institute, Zhejiang Cancer Hospital, Hangzhou, Zhejiang 310021, P.R. China
\end{abstract}

Received November 17, 2013; Accepted June 19, 2014

DOI: $10.3892 / 01.2014 .2386$

\begin{abstract}
Epidermal growth factor receptor (EGFR) is an important therapeutic target in lung cancer. Gefitinib and erlotinib, two reversible EGFR receptor tyrosine kinases inhibitors (TKIs), have been approved for the treatment of patients with metastatic non small-cell lung cancer. Icotinib, which is a selective EGFR-TKI, provides a similar efficacy to gefitinib. The present study aimed to investigate the survival and safety of icotinib in patients with lung adenocarcinoma with a poor performance status (PS). A total of 42 cases of lung adenocarcinoma, including 35 females and 7 males, were enrolled. Icotinib was used as the first-line of treatment due to poor PS of the patient or a more advanced age. Icotinib (125 mg) was orally administered three times per day. The overall response rate and disease control rates were 33.3 and $85.7 \%$, respectively. The median survival time was 13.0 months (95\% CI, 5.6-20.4), The median progression-free survival time was 7.0 months, and the 1-year survival rate was $71.4 \%$. A total of $79 \%$ of patients had an improved PS following icotinib treatment. Grade 1 to 2 rashes and diarrhea were the most frequent side effects. One patient succumbed during the study due to interstitial pneumonia. In conclusion, this is the first study indicating that patients with lung adenocarcinoma and poor PS may benefit from first-line icotinib therapy, but should be cautious of the occurrence of interstitial lung disease.
\end{abstract}

Correspondence to: Dr Meiyu Fang, Department of Integrated Chinese Traditional Medicine and Western Medicine, Zhejiang Cancer Hospital, 38 Guangji Road, Banshanqiao, Hangzhou, Zhejiang 310021, P.R. China

E-mail: fangjade2004@icloud.com

${ }^{*}$ Contributed equally

Key words: non-small cell lung cancer, adenocarcinoma, icotinib hydrochloride, performance status

\section{Introduction}

Lung cancer has the highest mortality rate of all cancers worldwide (1). A total of 70-75\% of all lung cancers are non small-cell lung cancer (NSCLC) with two-thirds presenting with locally advanced or metastatic disease at diagnosis. Treatment for these patients includes chemotherapy, radiotherapy and best supportive care (BSC) (2). Numerous efforts have been made to improve the treatment efficacy for advanced NSCLC.

Receptor tyrosine kinases, a family of transmembrane proteins, are important factors in cell signal transduction. These kinases control growth factor signal transmission from the cell surface to intracellular processes, and administrate critical cellular activities such as growth, differentiation, angiogenesis and inhibition of apoptosis. These signaling pathways promote the proliferation and formation of metastases of malignant cells. The epidermal growth factor receptor (EGFR) tyrosine kinase family is part of this family of receptor tyrosine kinases (3). Gefitinib and erlotinib are small-molecule tyrosine kinase inhibitors (TKIs) that target EGFR, and such inhibitors were the first targeted drugs to enter clinical use for the treatment of lung cancer $(4,5)$. These two drugs are the standard first-line treatment for patients with advanced NSCLC whose tumors have activating EGFR mutations. This treatment option has been associated with prolonged progression-free survival and improved tolerability and health-related quality of life, as compared with platinum-based doublet chemotherapy $(6,7)$.

Icotinib hydrochloride (BPI-2009H), an orally active, EGFR-TKI, has shown similar antitumor activity to gefitinib and erlotinib in patients with advanced NSCLC $(8,9)$. Based on preclinical and clinical data, icotinib has been shown to inhibit the growth of human tumor cell lines that over express EGFR and has a high level of tolerance among healthy Chinese subjects (10).

As the toxicity of TKIs is less than that of cytotoxic agents, their utility as a first-line treatment for patients with NSCLC with poor PS has been studied. Patients of East-Asian origin with adenocarcinoma have been shown to be significantly associated with a favorable response to EGFR TKIs $(4,5)$.

The present study proposed that icotinib would confer a survival advantage as a first-line therapy, compared with BSC, if eligible patients were selected on the basis of their histology. This retrospective study was conducted to evaluate the efficacy, toxicity and feasibility of first-line icotinib treatment 
for patients with adenocarcinoma of the lung together with extremely poor PS, who would not be eligible candidates for standard therapy.

\section{Materials and methods}

Patients. The medical charts of all patients with adenocarcinoma of the lung who received icotinib from May 1, 2011 to October 31, 2012 at the Zhejiang Cancer Hospital (Hangzhou, China), were reviewed. Of the 174 lung adenocarcinoma patients treated with icotinib, 42 cases were treated as first-line due to poor PS, with no indication for routine therapy such as surgical intervention, chemotherapy or radiotherapy. The patients were aged from 35 to 85 years, with a median age of 62.5 years. Each patient was evaluated, which included clinical history and physical examination, computed tomography (CT) of the chest, hematology and blood chemistry profiles prior to treatment. The study was approved by the ethics committee of Zhejiang Cancer Hospital.

Pathological analysis. Lung adenocarcinoma was confirmed either histologically or cytologically. Cytological specimens were obtained from the sputum, bronchial biopsy, pleural effusion and needle aspiration biopsy. Mutations in the extracted DNA of eight specimens from 42 NSCLC patients were examined by polymerase chain reaction-based direct sequencing for EGFR (exons 19 and 21).

Drug administration. Icotinib (125 mg) was orally administered three times per day (patent no. WO2003082830; Zhejiang Bata Pharma Ltd., Hangzhou, China). Tablets were taken $1 \mathrm{~h}$ before or after eating until disease progression or undue toxicity was observed. For patients with severe toxicity, the icotinib dosing schedule could be decreased to twice per day. Second-line chemotherapy or other treatments following the termination of icotinib therapy were permitted.

Clinical assessment. The objective tumor responses were evaluated as the complete response (CR), partial response (PR), stable disease (SD) or progressive disease (PD), in accordance with the Response Evaluation Criteria in Solid Tumors (11). Disease control was defined as the complete response + partial response + stable disease, which was confirmed and sustained for 4 weeks or longer. Baseline assessments were performed within 28 days of commencement of the treatment. Assessments were performed every 4 weeks for the first 4 months and then every 8 weeks until disease progression. All adverse events were reported and graded according to the National Cancer Institute Common Toxicity Criteria (version 3.0) (12). Data were also collected when icotinib treatment was interrupted or withdrawn due to adverse events. Changes in the PS scores during the course of the treatment were compared with the baseline score. Routine clinical and laboratory assessments were performed at least every 4 weeks.

Statistical analyses. The primary endpoint was to evaluate the progression-free survival (PFS). PFS was defined as the interval between the start of the treatment and the date of the first observation of disease progression or death from any cause. Secondary endpoints were the overall survival and response rate. Overall survival (OS) was assessed from the
Table I. Patient characteristics.

\begin{tabular}{lc}
\hline Characteristic & $\mathrm{n}(\%)$ \\
\hline Age, years & \\
Gender & $62.5(35-85)$ \\
Male & \\
Female & $7(16.7)$ \\
ECOG performance status & $35(83.3)$ \\
$0-1$ & \\
2 & $1(2.4)$ \\
$3-4$ & $9(21.4)$ \\
Smoking status, pack-years & $32(76.2)$ \\
0 & \\
1-19 & $34(84.0)$ \\
$\geq 20$ & $3(7.1)$ \\
Stage & $5(11.9)$ \\
I & \\
II & $1(2.4)$ \\
III & $1(2.4)$ \\
IV & $5(11.9)$ \\
Metastatic site & $35(83.3)$ \\
Lung & \\
Bone & $13(31.0)$ \\
Liver & $14(33.3)$ \\
Brain & $8(19.0)$ \\
Malignant pleural effusion & $5(11.9)$ \\
Other & $7(16.7)$ \\
EGFR & $4(9.5)$ \\
Mutation & \\
Wild type & $2(4.8)$ \\
Unknown & $6(14.3)$ \\
& $34(81.0)$ \\
\hline
\end{tabular}

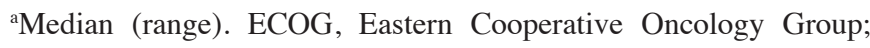
EGFR, epidermal growth factor receptor.

date of icotinib treatment until death from any cause. PFS and OS were estimated using the Kaplan-Meier method, and the differences between subgroups were analyzed by the log-rank statistic. The strata were smoking history, performance status, and gender. Tertiary endpoints were PS improvement rate and toxicity. $\mathrm{P}<0.05$ was considered to indicate a statistically significant difference. SPSS, version 13.0 (SPSS, Inc., Chicago, IL, USA) was used for statistical analyses.

\section{Results}

Patient characteristics. A total of 510 patients with NSCLC were screened between May 1, 2011 and October 31, 2012. Among them, 174 lung adenocarcinoma patients were treated with icotinib, and 42 patients with poor PS entered this study (Table I). The majority of patients had stage IV disease, and 
Table II. Patient responses to treatment.

\begin{tabular}{lcc}
\hline Response & No. of patients & Response rate, \% \\
\hline Complete response & 0 & 0 \\
Partial response & 14 & 33.3 \\
Stable disease & 22 & 52.4 \\
Progressive disease & 6 & 13.3 \\
Overall response & 14 & 33.3 \\
Disease control rate & 36 & 85.7 \\
\hline
\end{tabular}

Table III. Toxicities related to the treatment.

\begin{tabular}{lllllr}
\hline & \multicolumn{4}{c}{ Grade } & Total \\
\cline { 2 - 5 } Toxicity & 1 & 2 & 3 & 4 & no., \% \\
\hline Rash & 8 & 5 & 2 & 0 & 35.7 \\
Dry skin & 9 & 6 & 1 & 0 & 38.1 \\
Mucositis & 1 & 3 & 0 & 0 & 9.5 \\
Anorexia & 2 & 2 & 0 & 0 & 9.5 \\
Fatigue & 5 & 1 & 0 & 0 & 14.3 \\
Diarrhea & 8 & 7 & 3 & 1 & 45.2 \\
Vomiting & 2 & 1 & 0 & 0 & 7.1 \\
Lung & 0 & 0 & 0 & 1 & 2.3 \\
Increased ALT & 2 & 1 & 0 & 0 & 7.1 \\
\hline
\end{tabular}

ALT, alanine transaminase.

11/42 (26.2\%) patients had multiple sites of distant metastases. Thirty-two patients had Eastern Cooperative Oncology Group (ECOG) PS 3 or 4 due to various cancer-related conditions, including respiratory failure owing to multiple pulmonary metastasis, carcinomatous lymphangiosis, malignant pleural effusion and oxygen dependence. A total of 32 patients, $<75$ years old, had ECOG PS 3 to 4; nine cases, 75 to 79 years-of-age, had ECOG PS 2; and one patient, 80-years-old, had ECOG PS 1 . Heavy smokers (defined as $>10$ pack-years) were included in the study, although the majority of patients with adenocarcinomas were non- or light-smokers. EGFR mutation analysis was performed on eight patients before the treatment, of which two had an exon 19 deletion, and one had an L858R mutation.

Response and survival. The objective tumor responses are summarized in Table II. The overall response (OR) and disease control rates were 33.3 and $85.7 \%$, respectively. Stratified analyses were performed to examine the differences in response rate between certain clinical factors. The analyses revealed that gender, smoking status and PS had no association with icotinib response. For the two patients who had EGFR mutations, both achieved PR and longer PFS of more than 12 months, which were significantly more effective as compared with those with wild-type EGFR (where only 50\% achieved SD and the median PFS was seven months).

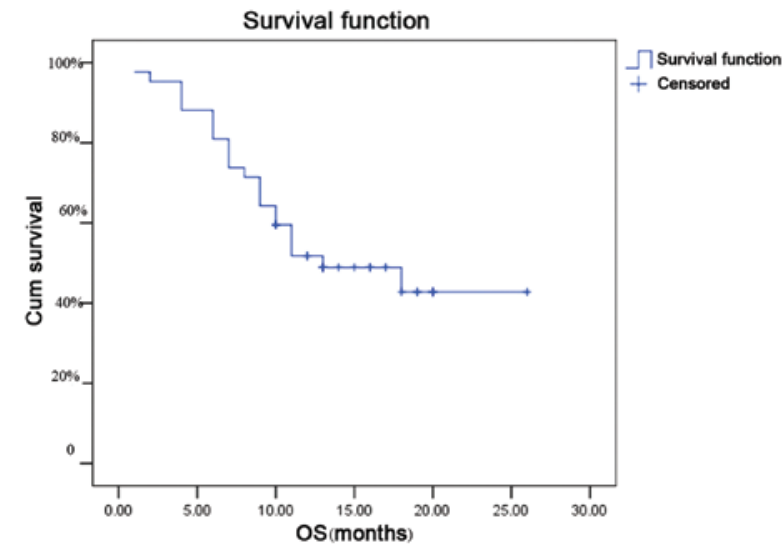

Figure 1. Kaplan-Meier estimates of OS for all patients from start of treatment. The median OS time was 13.0 months. Crosses indicate censored data. OS, overall survival; Cum survival, cumulative survival.

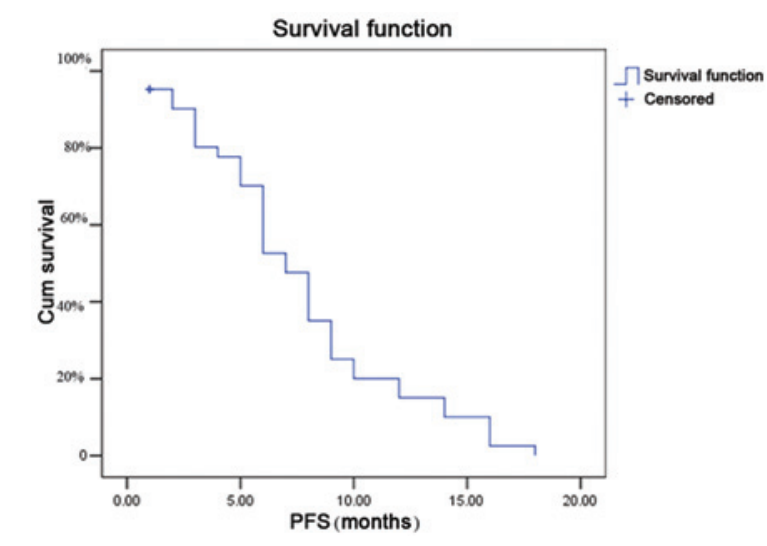

Figure 2. Kaplan-Meier estimates of PFS. The median PFS time was 7.0 months. Crosses indicate censored data. PFS, progression-free survival; Cum survival, cumulative survival.

The follow-up time was 12 to 26 months (median, 17.8 months), and 20/42 patients were alive on the last day of the follow up. The median survival time was 13.0 months (95\% CI, 5.6-20.4; Fig. 1), the median PFS time was 7.0 months (Fig. 2) and the 1-year survival rate was $71.4 \%$.

Improvement of PS. Thirty-three (79\%) of 42 patients had a significant improvement in their PS following icotinib treatment (Wilcoxon signed rank test, $\mathrm{P}=0.005$ ).

Toxicity. The toxicities that occurred during the icotinib treatment are listed in Table III. The majority of the adverse events were grade 1 or 2 . The most frequent adverse events were rash, diarrhea and fatigue. Two patients had grade 2 or worse AST/ALT elevation and one patient died of grade 4 interstitial lung disease. No hematological toxicity was observed in any of the patients. Reduction of the dosage or discontinuation of icotinib was not required in any of the patients.

\section{Discussion}

The lack of effective therapies in patients with advanced NSCLC and extremely poor PS (particularly PS 3-4) is a major clinical 
problem. EGFR gene family members have been shown to be widely expressed in various human cancers, including breast, head and neck, NSCLC and ovarian cancers (1). Gefitinib is a highly specific EGFR kinase inhibitor. However, no clinical benefit was identified in a randomized trial comparing gefitinib with BSC for unselected patients with NSCLC exhibiting poor PS (13). By selecting patients with adenocarcinoma who had never smoked, the administration of gefitinib in patients with advanced or metastatic NSCLC had a marked OR of $\sim 55.6 \%$ (14), which was comparable to gefitinib for EGFR mutation-positive patients with PS 0-2 (15-20).

Icotinib is a small-molecule inhibitor of EGFR tyrosine kinase, with a similar chemical structure and active mechanism to gefitinib (9), and was recently approved by the State Food and Drug Administration of China (http://app1.sfda.gov.cn/datasearch/face3/base.jsptatement.). The current retrospective study demonstrated that oral administration of icotinib was well tolerated in patients with lung adenocarcinoma with a poor PS. The median PFS time (7.0 months) was markedly improved as compared with that expected in patients managed only with BSC (7). The PFS time reported in the present study was improved over that reported previously for unselected patients undergoing standard chemotherapy with PS 2 treated with erlotinib (median PFS time, 1.9 to 2.9 months). Only a few male patients were enrolled in this study; therefore, precaution should be taken in interpreting this data, since female patients have been shown to survive longer than male patients $(21,22)$.

In the present study, two patients with EGFR exon 19 or 21 mutations had a PR. This data is inconsistent with the majority of previous studies $(6,7)$; however, the sample numbers were low due to poor PS or older age of the patients, though the assessment of EGFR mutation status before treatment is considered to be reasonable and predictive.

In the present study, the most frequent toxicities were rashes and diarrhea, both of which were grade 1 and 2 . One patient, however, succummbed to uncontrollable severe interstitial pneumonia.

In conclusion, icotinib is active in Chinese patients with lung adenocarcinoma with poor PS. Data from the present study suggests that icotinib should not be used in patients with interstitial lung disease or concurrent with uncontrolled pneumonia. Further randomized trials are required to delineate the role of icotinib as a first-line treatment in this subset of selected patients as compared with BSC or other TKIs.

\section{Acknowledgements}

The present study was supported by the Medical Scientific Research Foundation of Zhejiang Province of China (grant no. 2013KYB051).

\section{References}

1. Jemal A, Bray F, Center MM, et al: Global cancer statistics. CA. Cancer J Clin 61: 69-90, 2011.

2. Fang M, Wang S, Zheng Y, et al: Maintenance therapy with oral etoposide following first-line docetaxel-cisplatin chemotherapy in metastatic non-small cell lung cancer patients. Bangladesh J Pharmacol 7: 192-198, 2012.

3. Sharma S, Bell D, Settleman J and Haber DA: Epidermal growth factor receptor mutations in lung cancer. Nat Rev Cancer 7:169-181, 2007.
4. Fukuoka M, Yano S, Giaccone G, et al: Multi-institutional randomized phase II trial of gefitinib for previously treated patients with advanced non-small-cell lung cancer (The IDEAL 1 Trial). J Clin Oncol 21: 2237-2246, 2003.

5. Kris MG, Natale RB, Herbst RS, et al: Efficacy of gefitinib, an inhibitor of the epidermal growth factor receptor tyrosine kinase, in symptomatic patients with non-small cell lung cancer: a randomized trial. JAMA 290: 2149-2158, 2003.

6. Maemondo M, Inoue A, Kobayashi K, et al: Gefitinib or chemotherapy for non-small-cell lung cancer with mutated EGFR. N Engl J Med 362: 2380-2388, 2010.

7. Mitsudomi T, Morita S, Yatabe Y, et al: Gefitinib versus cisplatin plus docetaxel in patients with non-small-cell lung cancer harbouring mutations of the epidermal growth factor receptor (WJTOG3405): an open label, randomised phase 3 trial. Lancet Oncol 11: 121-128, 2010.

8. Zhao Q, Shentu J, Xu N, et al: Phase I study of icotinib hydrochloride (BPI-2009H), an oral EGFR tyrosine kinase inhibitor, in patients with advanced NSCLC and other solid tumors. Lung Cancer 73: 195-202, 2011.

9. Tan F, Shen X, Wang D, et al: Icotinib (BPI-2009H), a novel EGFR tyrosine kinase inhibitor, displays potent efficacy in preclinical studies. Lung Cancer 76: 177-182, 2012.

10. Liu D, Jiang J, Hu P, et al: Quantitative determination of icotinib in human plasma and urine using liquid chromatography coupled to tandem mass spectrometry. J Chromatogr B Analyt Technol Biomed Life Sci 877: 3781-3786, 2009.

11. Therasse P, Arbuck SG, Eisenhauer EA, et $a$ : New guidelines to evaluate the response to treatment in solid tumors. European Organization for Research and Treatment of Cancer, National Cancer Institute of the United States, National Cancer Institute of Canada. J Natl Cancer Inst 92: 205-216, 2000.

12. National Cancer Institute.National Cancer Institute Common Toxicity Criteria.http://ctep.cancer.gov/protocolDevelopment/electronic.applications/docs/ctcaev3.pdf. Accessed June 22, 2014.

13. Thatcher N, Chang A, Parikh P, et al: Gefitinib plus best supportive care in previously treated patients with refractory advanced non-small-cell lung cancer: results from a randomised, placebo-controlled, multicentre study (Iressa Survival Evaluation in Lung Cancer). Lancet 366: 1527-1537, 2005.

14. Lee DH, Han JY, Yu SY, et al: The role of gefitinib treatment for Korean never-smokers with advanced or metastatic adenocarcinoma of the lung: a prospective study. J Thorac Oncol 1: 965-971, 2006.

15. Inoue A, Suzuki T, Fukuhara T, et al: Prospective phase II study of gefitinib for chemotherapy-naive patients with advanced non-small-cell lung cancer with epidermal growth factor receptor gene mutations. J Clin Oncol 24: 3340-3346, 2006.

16. Asahina H, Yamazaki K, Kinoshita I, et al: A phase II trial of gefitinib as first-line therapy for advanced non-small cell lung cancer with epidermal growth factor receptor mutations. Br J Cancer 95: 998-1004, 2006.

17. Sunaga N, Tomizawa Y, Yanagitani N, et al: Phase II prospective study of the efficacy of gefitinib for the treatment of stage III/IV non-small cell lung cancer with EGFR mutations, irrespective of previous chemotherapy. Lung Cancer 56: 383-389, 2007.

18. Sutani A, Nagai Y, Udagawa K, et al: Gefitinib for non-small-cell lung cancer patients with epidermal growth factor receptor gene mutations screened by peptide nucleic acid-locked nucleic acid PCR clamp. Br J Cancer 95: 1483-1489, 2006.

19. Paz-Ares L, Sanchez JM, García-Velasco A, et al: A prospective phase II trial of erlotinib in advanced non-small cell lung cancer (NSCLC) patients (p) with mutations in the tyrosine kinase (TK) domain of the epidermal growth factor receptor (EGFR). J Clin Oncol 24: 369s, 2006.

20. Sequist LV, Martins RG, Spigel D, et al: iTARGET: A phase II trial to assess the response to gefitinib in epidermal growth factor receptor $(E G F R)$-mutated non-small cell lung cancer (NSCLC) tumors. J Clin Oncol 25: 386s, 2007.

21. O'Connell J, Kris MG, Gralla RJ, et al: Frequency and prognostic importance of pretreatment clinical characteristics in patients with advanced non-small cell lung cancer treated with combination chemotherapy. J Clin Oncol 4: 1604-1614, 1986.

22. Radzikowska E, Glaz P and Roszkowski K: Lung cancer in women: age, smoking, histology, performance status, stage, initial treatment, and survival. Population-based study of 20561 cases. Ann Oncol 13: 1087-1093, 2002. 